\title{
Pulse Pressure Analysis to Guide Intraoperative Phlebotomy Prior to Cardiac Surgery
}

\author{
Brian Schloss ${ }^{\text {a, c }}$, Dmitry Tumin ${ }^{\mathrm{a}}$, Aymen Naguib ${ }^{\mathrm{a}}$, Julie Rice ${ }^{\mathrm{a}}$, \\ Mark Galantowicz ${ }^{\mathrm{b}}$, Joseph D. Tobias ${ }^{\mathrm{a}}$
}

\begin{abstract}
Background: The aim of this study was to evaluate the response of the LiDCO-rapid ${ }^{\mathrm{TM}}$ during intraoperative phlebotomy in anesthetized children prior to surgery for congenital heart disease.

Methods: After the induction of general anesthesia and endotracheal intubation, baseline vital signs were recorded, along with pulse pressure variability (PPV) and stroke volume variability (SVV) from the LiDCO-rapid ${ }^{\mathrm{TM}}$ and cerebral oxygenation $\left(\mathrm{rSO}_{2}\right)$ using near-infrared spectroscopy (NIRS). Phlebotomy was performed over 5 - $10 \mathrm{~min}$ with the volume of blood removed calculated to achieve a hematocrit of $24-28 \%$ on cardiopulmonary bypass. The primary outcome was a decline in $\mathrm{rSO}_{2} \geq 5$ between the baseline value and the end of phlebotomy. At that time, the correlation of the starting and ending values of SVV and PPV with the NIRS was determined.
\end{abstract}

Results: The study cohort included 30 patients (mean age of $21 \pm 11$ years). Statistically significant changes during the study period were observed in $\mathrm{rSO}_{2}$, but not in the LiDCO-rapid ${ }^{\mathrm{TM}}$ parameters. In analysis of continuous NIRS data, the change in NIRS did not correlate with either baseline $(r=0.10, P=0.644)$ or final $(r=0.02, P=0.914)$ SVV. Likewise, the change in NIRS did not correlate with baseline $(\mathrm{r}$ $=0.01, \mathrm{P}=0.953)$ or final $(\mathrm{r}=0.00, \mathrm{P}=0.982) \mathrm{PPV}$.

Conclusion: Baseline values as well as changes in the PVV and SVV from the LiDCO-rapid ${ }^{\mathrm{TM}}$ did not predict or correlate with changes in cerebral oxygenation measured by NIRS during intraoperative phlebotomy. Our preliminary data suggest that these parameters (PVV and SVV) are not useful in monitoring patient stability or the need for volume replacement during intraoperative phlebotomy prior to cardiac surgery.

Keywords: Cardiac surgery; Pulse pressure variation

Manuscript submitted November 22, 2017, accepted November 28, 2017

aDepartment of Anesthesiology \& Pain Medicine, Nationwide Children's Hospital, Columbus, $\mathrm{OH}$, USA

bDepartment of Pediatric Cardiothoracic Surgery, Nationwide Children's Hospital, Columbus, $\mathrm{OH}$, USA

${ }^{\mathrm{c} C o r r e s p o n d i n g ~ A u t h o r: ~ B r i a n ~ S c h l o s s, ~ D e p a r t m e n t ~ o f ~ A n e s t h e s i o l o g y ~ \& ~ P a i n ~}$ Medicine, Nationwide Children's Hospital, 700 Children's Drive, Columbus, OH 43205, USA. Email: Brian.Schloss@Nationwidechildrens.org

doi: https://doi.org/10.14740/cr634w

\section{Introduction}

Recent investigations have demonstrated the potential risks associated with the administration of allogeneic blood products. Given these considerations, various techniques have been implemented perioperatively to limit the need for allogeneic blood products [1,2]. One such technique, acute normovolemic hemodilution (ANH), involves the intraoperative removal of whole blood from the patient prior to surgical incision, so that it can be re-infused postoperatively. The removed blood is generally replaced by a 3:1 ratio of crystalloid or 1:1 ratio of colloid to maintain euvolemia. Rather than utilizing an arbitrary ratio for volume replacement during the phlebotomy phase of ANH, our practice during surgery for congenital heart disease (CHD) with the use of cardiopulmonary bypass (CPB) is to administer the minimum necessary volume to maintain hemodynamic stability, as the secondary hemodilution which will occur due to the crystalloid prime of CPB may result in an unacceptably low hematocrit [3-5]. During the process of intraoperative phlebotomy, various parameters can be followed to assess the patient's stability, the need for volume administration to replace the phlebotomized blood, or the need to stop blood withdrawal. Hemodynamic stability may be assessed using changes in heart rate (HR), the mean arterial pressure (MAP), and the electrocardiogram (ECG), whereas end-organ perfusion is assessed using cerebral oxygenation $\left(\mathrm{rSO}_{2}\right)$ measured by near-infrared spectroscopy (NIRS) $[3,4]$.

During non-cardiac surgery, dynamic parameters of fluid responsiveness, such as pulse pressure variation (PPV) and stroke volume variation (SVV), have been shown to reflect hemodynamic changes induced by intermittent positive pressure ventilation $[6,7]$. These indices have proven to be superior to static measures of preload in predicting stroke volume increases in response to fluid challenges. Furthermore, the use of these dynamic parameters in a goal-directed fashion has been shown to improve outcomes in high risk adult surgical patients $[8,9]$. The LiDCO-rapid ${ }^{\mathrm{TM}}$ (LiDCO, Cambridge, UK) is an uncalibrated pulse contour analysis algorithm system. It provides beat to beat reporting of PPV as well as estimates of stroke volume and SVV. To date, this technology has not been applied as a means of assessing hemodynamic stability during intraoperative phlebotomy as part of an ANH protocol in cardiac surgery. In the current study, we compared changes in PPV and SVV to changes in NIRS during the removal of blood prior to $\mathrm{CPB}$ and surgery for CHD. We hypothesized that PPV 
and SVV values exceeding 13\% would demonstrate agreement with declines of at least $5 \%$ or $10 \%$ in $\mathrm{rSO}_{2}$ on the NIRS.

\section{Methods}

The study was approved by the institutional review board at Nationwide Children's Hospital. Over a 12-month period, data were collected prospectively on patients who were scheduled for surgery for CHD in whom intraoperative phlebotomy was planned as part of an ANH protocol for blood conservation. After the induction of general anesthesia, endotracheal intubation and mechanical ventilation with standard monitoring according to the standards of the American Society of Anesthesiologists, a peripheral arterial cannula was placed and the LiDCO-rapid monitor was attached. The NIRS monitor (INVOSTM 5100C Cerebral Oximeter, Covidien/Medtronic, Minneapolis, MN, USA) was placed on the patient's forehead using an age and weight-appropriate probe. Anesthesia was maintained with isoflurane or sevoflurane in $100 \%$ oxygen. During a period of hemodynamic stability, baseline vital signs and ventilator settings were recorded, along with PPV and $\mathrm{SVV}$ from the LiDCO ${ }^{\mathrm{TM}}$-rapid, and $\mathrm{rSO}_{2}$ from the NIRS. Phlebotomy was then performed over $5-10 \mathrm{~min}$. The volume of blood removed was calculated based on the patient's weight, starting hematocrit, and a target hematocrit after the initiation of CPB of 24-28\%. Data were recorded again at the conclusion of phlebotomy. Based on pre-phlebotomy calculation as well as hemodynamic response, not all patients had a total of $10 \mathrm{~mL} / \mathrm{kg}$ of blood removed. During phlebotomy, vital signs and NIRS readings were monitored continuously by the anesthesia team. Changes of vital signs by more than $20 \%$ from baseline or a decrease in NIRS by more than 10 were treated by the administration of volume replacement with crystalloid, the administration of a vasoactive agent (phenylephrine), or temporary cessation of phlebotomy. These interventions were at the discretion of the anesthesiologist. No interventions were initiated on the basis of changes in SVV or PPV from the LiDCO-rapid ${ }^{\mathrm{TM}}$.

The primary outcome was a decline in $\mathrm{rSO}_{2} \geq 5$ between the baseline value at the start of phlebotomy and the end of phlebotomy. This threshold was selected as the minimal clinically significant difference in the $\mathrm{rSO}_{2}$ value based on our usual clinical practice [3]. A greater decline in $\mathrm{rSO}_{2}(\geq 10)$ was considered as a secondary outcome. Cases were further categorized according to whether PPV and SVV values were $\geq$ $13 \%$ at the baseline or the final measurement. The threshold of $13 \%$ was chosen based on previous literature suggesting reliable prediction of fluid responsiveness beyond this threshold of variation [10]. Agreement between each PPV and SVV threshold and the two thresholds for $\mathrm{rSO}_{2}$ change was determined using Cohen's kappa statistic. In further analysis, continuous data on changes in $\mathrm{rSO}_{2}$ and $\mathrm{LiDCO}^{\mathrm{TM}}$-rapid measurements over the course of the study were characterized using paired $t$-tests, and the change in $\mathrm{rSO}_{2}$ was correlated with baseline and final $\mathrm{LiDCO}^{\mathrm{TM}}$-rapid measurements using Pearson's correlation coefficient. Due to the exploratory nature of this study, no adjustments for multiple comparisons were performed, and
Table 1. Study Cohort Characteristics $(N=30)$

\begin{tabular}{ll}
\hline Variable & Mean (SD) or N (\%) \\
\hline Age (years) & $22(11)$ \\
Male gender & $15(50 \%)$ \\
Height $(\mathrm{cm})$ & $169(11)$ \\
\hline Weight $(\mathrm{kg})$ & $71(22)$ \\
Body mass index $\left(\mathrm{kg} / \mathrm{m}^{2}\right)$ & $24(5)$ \\
Surgical procedure & \\
$\quad$ Pulmonary valve replacement & $6(20 \%)$ \\
\multicolumn{1}{l}{ Aortic valve replacement } & $4(13 \%)$ \\
$\quad$ Tricuspid valve replacement & $3(10 \%)$ \\
\multicolumn{1}{c}{ Other } & $17(57 \%)$ \\
Tidal volume (mL/kg) & $7.6(2.1)$ \\
Total blood withdrawn $(\mathrm{mL} / \mathrm{kg})$ & $8.6(2.9)$ \\
\hline
\end{tabular}

aperformed with aortoplasty ( $n=2)$; with right ventricle to pulmonary artery conduit repair $(n=1)$.

no a priori sample size calculation was undertaken. Enrollment was targeted for 30 patients according to the feasibility of completing data collection in the planned study period. Data analysis was performed in Stata/IC 13.1 (StataCorp, LP, College Station, TX, USA), and $\mathrm{P}<0.05$ was considered statistically significant.

\section{Results}

The study cohort included a total of 30 patients (15/15 male/ female, age $21 \pm 11$ years). Of these 30 patients, 27 had NIRS data recorded at baseline and the end of phlebotomy. The characteristics of the study sample are summarized in Table 1 and the data obtained at each time point are summarized in Table 2. Statistically significant changes during the study period were observed in $\mathrm{rSO}_{2}$, but not in the $\mathrm{LiDCO}^{\mathrm{TM}_{\text {-rapid }}}$ parameters of $\mathrm{SVV}$ and PPV. The average decline in $\mathrm{rSO}_{2}$ on the NIRS was $5 \pm 7 \%$, with 17 of 27 patients experiencing a $\geq 5$ decline and five of 27 patients experiencing a $\geq 10$ decline. Among the 23 patients with baseline $\mathrm{LiDCO}^{\mathrm{TM}}$-rapid data, six had an SVV $\geq$ $13 \%$ and 14 had a PPV $\geq 13 \%$. Using baseline LiDCO-rapid measurements, SVV $\geq 13 \%$ correctly classified two of 14 cases with an NIRS decline $\geq 5$ and five of nine cases with an NIRS decline $<5$ (30\% agreement; $\kappa=-0.26 ; \mathrm{P}=0.946)$. Baseline PPV $\geq 13 \%$ correctly classified nine of 14 cases with an NIRS decline $\geq 5$, and four of nine cases with an NIRS decline $<5$ (57\% agreement; $\kappa=0.09 ; \mathrm{P}=0.338)$. At the higher threshold of $\mathrm{rSO}_{2}$ decline, baseline $\mathrm{SVV} \geq 13 \%$ correctly classified one of five cases with an NIRS decline $\geq 10$ and 13 of 18 cases with an NIRS decline $<10 \%$ (61\% agreement; $\kappa=-0.07 ; \mathrm{P}=0.637)$, whereas baseline PPV $\geq 13 \%$ correctly classified three of five cases with an NIRS decline $\geq 10$ and seven of 18 patients with an NIRS decline $<10$ (43\% agreement; $\kappa=-0.01 ; \mathrm{P}=0.518)$.

Among the 25 patients with $\mathrm{LiDCO}^{\mathrm{TM}}$-rapid data at the final time point, eight had an SVV $\geq 13 \%$ and 18 had a PPV $\geq 13 \%$. Using the final LiDCO ${ }^{\mathrm{TM}}$-rapid measurements, SVV 
Table 2. LiDCO ${ }^{\mathrm{TM}}$-Rapid and Cerebral Oxygenation During the Study Protocol

\begin{tabular}{|c|c|c|c|c|}
\hline \multirow{2}{*}{ Variable } & \multirow{2}{*}{ Baseline, mean (SD) } & \multirow{2}{*}{ End of phlebotomy, mean (SD) } & \multicolumn{2}{|c|}{ Change from baseline to end of phlebotomy } \\
\hline & & & Difference in means $(95 \% \mathrm{CI})$ & P-value \\
\hline NIRS $\mathrm{rSO}_{2}{ }^{\mathrm{a}}$ & $74(7)$ & $69(8)$ & $-5(-2,-8)$ & $<0.001$ \\
\hline SVV\% & $11(6)$ & $12(6)$ & $2(-1,5)$ & 0.143 \\
\hline PPV\% & $17(12)$ & $17(7)$ & $1(-4,6)$ & 0.624 \\
\hline
\end{tabular}

aData missing in three cases at baseline. 'bata missing in four cases at baseline and two cases at end of phlebotomy. NIRS: near-infrared spectroscopy; $\mathrm{rSO}_{2}$ : cerebral oxygenation; PPV: pulse pressure variability; SVV: stroke volume variability.

$\geq 13 \%$ correctly classified three of 16 cases with an NIRS decline $\geq 5$, and four of nine cases with an NIRS decline $<5$ $(28 \%$ agreement; $\kappa=-0.31 ; \mathrm{P}=0.971)$. Final PPV $\geq 13 \%$ correctly classified 11 of 16 cases with an NIRS decline $\geq 5$, and two of nine cases with an NIRS decline $<5$ (52\% agreement; $\kappa=-0.09 ; \mathrm{P}=0.685)$. Final $\mathrm{SVV} \geq 13 \%$ correctly classified two of five cases with an NIRS decline $\geq 10$, and 14 of 20 cases with an NIRS decline $<10(64 \%$ agreement; $\kappa=0.08$; $\mathrm{P}=0.334$ ). Final $\mathrm{PPV} \geq 13 \%$ correctly classified five of five cases with an NIRS decline $\geq 10$, and seven of 20 cases with an NIRS decline $<10$ (48\% agreement; $\kappa=0.18 ; \mathrm{P}=0.060)$. In analysis of continuous NIRS data, the change in NIRS was not correlated with either baseline $(\mathrm{r}=0.10, \mathrm{P}=0.644)$ or final $(\mathrm{r}=0.02, \mathrm{P}=0.914) \mathrm{SVV}$. Likewise, change in NIRS was not correlated with baseline $(\mathrm{r}=0.01 ; \mathrm{P}=0.953)$ or final $(\mathrm{r}=0.00$; $\mathrm{P}=0.982$ ) PPV.

\section{Discussion}

ANH is one method of limiting allogenic blood transfusion during major surgical procedures including surgery for CHD using CPB $[11,12]$. The NIRS monitor, which has been validated against cerebral tissue oxygenation and jugular venous bulb saturation, is able to non-invasively monitor cerebral venous oxygen saturation and provide an estimation of brain tissue oxygenation [13]. Our institution has previously shown that intraoperative phlebotomy during $\mathrm{ANH}$ and the need for volume replacement may be more appropriately guided by NIRS rather than traditional hemodynamic variables of HR and BP [3]. Changes in vital signs may occur early without changes in $\mathrm{rSO}_{2}$ as measured by NIRS, suggesting that cardiac output and tissue oxygenation are maintained despite changes in vital signs.

Dynamic parameters of fluid responsiveness such as PPV and SVV reflect changes in cardiac output induced by intermittent positive pressure ventilation [14]. As intravascular volume decreases, the normal variation in preload and hence cardiac output that occurs related to positive pressure ventilation is accentuated. These changes are noted as an increase in the PPV and SVV. These variables have been shown to be superior to static hemodynamic variables in predicting fluid responsiveness [6-9]. The current study evaluated the correlation of PPV and SVV values, measured by the LiDCO ${ }^{\mathrm{TM}}$-rapid, with changes in $\mathrm{rSO}_{2}$ from the NIRS, to evaluate the utility of the $\mathrm{LiDCO}^{\mathrm{TM}}$-rapid device in evaluating hemodynamic stability, guiding preoperative phlebotomy, and the need for volume replacement. Our preliminary data indicate that PPV and SVV values did not significantly change after phlebotomy, despite the decline in $\mathrm{rSO}_{2}$ noted on the NIRS. Furthermore, changes in the dynamic parameters did not correlate with declines in $\mathrm{rSO}_{2}$. Given that the blood withdrawal during preoperative phlebotomy would be expected to induce an acute state of intravascular volume depletion, we hypothesized that increases in PPV and SVV would occur. Furthermore, as these dynamic parameters are relatively rapid, we predicted that these changes would occur prior to changes in end-organ oxygenation including $\mathrm{rSO}_{2}$ on the NIRS. However, no such correlation or change was observed.

The reasons for the lack of predictive utility for PPV and SVV in this setting are not entirely clear. We speculate that the effects of general anesthesia including the volatile anesthetic agents, sevoflurane and isoflurane, may blunt the sympathetic hemodynamic response to ANH. The acute nature of the phlebotomy, blunting of the associated sympathetic response, and the baseline hemodynamic changes induced by general anesthesia may not allow the PPV and SVV to adequately reflect a fluid responsive state. Our patient cohort had a mean age of 20 years and therefore based on the increased elasticity of the vasculature, the changes in PPV and SVV may be different from that seen in the older adult population in whom other studies have been performed. Furthermore, the LiDCO ${ }^{\text {TM}}$ rapid and other pulse contour analyzing monitors, may not be suited to respond to rapid and acute changes in intravascular volume.

Limitations of the current study include that it was conducted at a single center, and was observational in nature without specific interventions required based on changes in SVV, $\mathrm{PVV}$ or $\mathrm{rSO}_{2}$. No protocols were used to standardize the phlebotomy and $\mathrm{ANH}$ process. The specific anesthetic technique was at the discretion of the attending anesthesiologist although it included a standard anesthetic technique for such procedures including fentanyl and a volatile anesthetic agent (sevoflurane or isoflurane). The specific ANH volumes varied from 5 to 10 $\mathrm{mL} / \mathrm{kg}$ in all of the patients, but were based on each patient's individual starting hematocrit as well as their hemodynamic stability during phlebotomy. The $\mathrm{LiDCO}^{\mathrm{TM}}$-rapid is an uncalibrated monitor whose calculations are proprietary. While PPV can be readily calculated from any arterial waveform, SVV calculation for this study was specific to the $\mathrm{LiDCO}^{\mathrm{TM}}$-rapid device and therefore cannot be readily extrapolated to other monitors of SVV. 
In conclusion, our preliminary data suggest that PPV and SVV are not useful in guiding intraoperative phlebotomy as part of our ANH protocol prior to the initiation of CPB. The dynamic parameters of SVV and PVV were not able to predict clinically significant declines in $\mathrm{rSO}_{2}$ measured by NIRS. While dynamic parameters have been shown to be useful to guide fluid therapy in major abdominal surgery in the older adult population, NIRS monitoring appears to be superior during ANH.

\section{References}

1. Lavoie J. Blood transfusion risks and alternative strategies in pediatric patients. Paediatr Anaesth. 2011;21(1):14-24.

2. Guzzetta NA. Benefits and risks of red blood cell transfusion in pediatric patients undergoing cardiac surgery. Paediatr Anaesth. 2011;21(5):504-511.

3. Dewhirst E, Winch P, Naguib A, Galantowicz M, Tobias JD. Cerebral oximetry monitoring during preoperative phlebotomy to limit allogeneic blood use in patients undergoing cardiac surgery. Pediatr Cardiol. 2013;34(1):7580 .

4. Schloss B, Naguib A, Bissonnette B, Winch P, Rice J, Miao Y, Tobias JD. Plethysmography variability index response to isovolemic hemodilution in children prior to surgery for congenital heart disease. J Pediatr Intensive Care. 2014;3:35-40.

5. Wypij D, Jonas RA, Bellinger DC, Del Nido PJ, Mayer JE, Jr., Bacha EA, Forbess JM, et al. The effect of hematocrit during hypothermic cardiopulmonary bypass in infant heart surgery: results from the combined Boston hematocrit trials. J Thorac Cardiovasc Surg. 2008;135(2):355360 .

6. Cavallaro F, Sandroni C, Antonelli M. Functional hemo- dynamic monitoring and dynamic indices of fluid responsiveness. Minerva Anestesiol. 2008;74(4):123-135.

7. Michard F, Teboul JL. Predicting fluid responsiveness in ICU patients: a critical analysis of the evidence. Chest. 2002;121(6):2000-2008.

8. Donati A, Loggi S, Preiser JC, Orsetti G, Munch C, Gabbanelli V, Pelaia P, et al. Goal-directed intraoperative therapy reduces morbidity and length of hospital stay in high-risk surgical patients. Chest. 2007;132(6):18171824.

9. Gan TJ, Soppitt A, Maroof M, el-Moalem H, Robertson KM, Moretti E, Dwane P, et al. Goal-directed intraoperative fluid administration reduces length of hospital stay after major surgery. Anesthesiology. 2002;97(4):820-826.

10. Cannesson M, Le Manach Y, Hofer CK, Goarin JP, Lehot JJ, Vallet B, Tavernier B. Assessing the diagnostic accuracy of pulse pressure variations for the prediction of fluid responsiveness: a "gray zone" approach. Anesthesiology. 2011;115(2):231-241.

11. Testa L, Tobias JD. Techniques of blood conservation (Part 1): Isovolemic hemodilution. Am J Anesthesiol. 1996;23:20-28.

12. Testa L, Tobias JD. Techniques of blood conservation (Part 2): Autologous transfusion therapy, cell saver devices, and pharmacologic control of erythropoiesis and coagulation. Am J Anesthesiol. 1996;23:63-72.

13. McLeod AD, Igielman F, Elwell C, Cope M, Smith M. Measuring cerebral oxygenation during normobaric hyperoxia: a comparison of tissue microprobes, near-infrared spectroscopy, and jugular venous oximetry in head injury. Anesth Analg. 2003;97(3):851-856.

14. Chung E, Cannesson M. Using non invasive dynamic parameters of fluid responsiveness in children: there is still much to learn. J Clin Monit Comput. 2012;26(3):153155. 